\title{
Publisher Correction: An integrated bacterial system for the discovery of chemical rescuers of disease-associated protein misfolding
}

Ilias Matis, Dafni Chrysanthi Delivoria, Barbara Mavroidi, Nikoletta Papaevgeniou, Stefania Panoutsou, Stamatia Bellou, Konstantinos D. Papavasileiou, Zacharoula I. Linardaki, Alexandra V. Stavropoulou, Kostas Vekrellis, Nikos Boukos, Fragiskos N. Kolisis, Efstathios S. Gonos, Marigoula Margarity, Manthos G. Papadopoulos, Spiros Efthimiopoulos, Maria Pelecanou, Niki Chondrogianni and Georgios Skretas

Correction to: Nature Biomedical Engineering https://doi.org/10.1038/s41551-017-0144-3 (2017); published online 10 October 2017.

In the version of this Article originally published, in Fig. 1c-e, on the $x$ axes, the lines labelled 'A $\beta_{42}$ ' and 'A $\beta_{42}(\mathrm{~F} 19 \mathrm{~S} ; \mathrm{L} 34 \mathrm{P})$ ' grouped the data incorrectly; the line labelled $\mathrm{A} \beta_{42}$ should have grouped the data for Random 1-2 and Clones 1-10, and the line labelled $\mathrm{A} \beta_{42}(\mathrm{~F} 19 \mathrm{~S} ; \mathrm{L} 34 \mathrm{P})$ should have only grouped the data for Random 1-2 on the right end of the plots and blots. These figures have now been corrected in all versions of the Article.

Published online: 2 January 2018

https://doi.org/10.1038/s41551-017-0164-z 